\title{
Searches for displaced jets and long-lived particles with ATLAS
}

\author{
Massimo Corradi* \\ INFN Bologna \\ E-mail: massimo.corradi@bo.infn.it
}

\section{On behalf of the ATLAS Collaboration}

The production of heavy long-lived particles in $p p$ collisions at LHC has been searched for with the ATLAS detector, exploiting experimental signatures such as vertices displaced from the $p p$ interaction point, slow charged particles traversing the detector, and energy deposits delayed with respect to the $p p$ interaction time. As no evidence for any excess above expected backgrounds has been found, new constraints on models of new physics have been set in the framework of gauge-mediated supersymmetry breaking models and of R-parity violating supersymmetry.

The European Physical Society Conference on High Energy Physics -EPS-HEP2013

18-24 July 2013

Stockholm, Sweden

\footnotetext{
* Speaker.
} 


\section{Introduction}

New physics beyond the Standard Model (SM) may appear in the form of long-lived particles (LLPs) with proper decay length $c \tau \geq 1 \mathrm{~mm}$. Examples of LLPs are found in several models. In gauge-mediated supersymmetry-breaking (GMSB) models the lightest supersymmetric particle (LSP) can be a gravitino and the next-to-lightest particle (NLSP) can be long-lived. In R-parity violating (RPV) supersymmetry, the LSP can be meta-stable, decaying into SM particles through the RPV couplings. Another class of models predicts the existence of an hidden sector, decoupled from the SM except for the interaction with the Higgs boson or other massive particles. Once produced, for example in Higgs boson decays, these hidden-sector particles would decay back to SM particles with long lifetimes.

This contribution summarizes the searches for LLPs performed with the ATLAS detector in $p p$ collisions at the LHC. Collider detectors such as ATLAS [1] are not optimized for identifying and measuring LLPs, as they are typically intended to measure SM particles produced close to the interaction point. These searches therefore involve an ongoing effort to improve the detector capability to detect these signals.

Depending on their lifetime, the LLPs would appear in the form of different experimental signatures. Several of these signatures have been searched for by ATLAS in different dedicated analyses. LLPs with $c \tau$ from $1 \mathrm{~mm}$ up to approximately $1 \mathrm{~m}$ could still decay inside the tracking Inner Detector (ID) and could be detected as a high-mass secondary vertex or as particles not pointing to the primary interaction. Two recent searches, one looking for non-pointing photons and one searching displaced vertices (DVs), are described in sections 目and 3, respectively. If the LLP is charged and decays into neutrals plus one charged track, it could be reconstructed as a track emerging from the $p p$ interaction and ending (or showing a "kink") at the decay point. The search for these disappearing tracks is reported in another contribution to these proceedings [2]. LLPs with longer lifetimes could decay mostly outside the ID volume. The ATLAS Muon Spectrometer (MS) can be used to reconstruct decay vertices at a distance of the order of $10 \mathrm{~m}$ from the interaction point. This capability was exploited in a dedicated search for neutral states produced in hiddensector models [邚.

Stable or very long-lived particles could traverse most of the ATLAS detector. In the case of charged particles they would be visible as penetrating tracks with high ionization and low velocity $\beta$. A new search for penetrating (meta-)stable sleptons in described in section $\sharp$. Multiply-charged particles and magnetic monopoles would also appear as highly-ionizing tracks and have been the subject of dedicated searches [4, 5]. Similar signatures have been also exploited in the search of long-lived squarks or gluinos in RPV models [6].

The LLPs could be stopped inside the detector material due to their large energy loss. If the lifetime is sufficiently long, their decays could be detected as energy deposits delayed with respect to $p p$ collision time. A new search for "out-of-time" jets, in the context of RPV models, is reported in section 5. Special trigger strategies, developed to select LLP signals, are described in [7].

\section{Non-pointing photons}

GMSB models in which the NLSP is the lightest neutralino decaying into a photon and a 


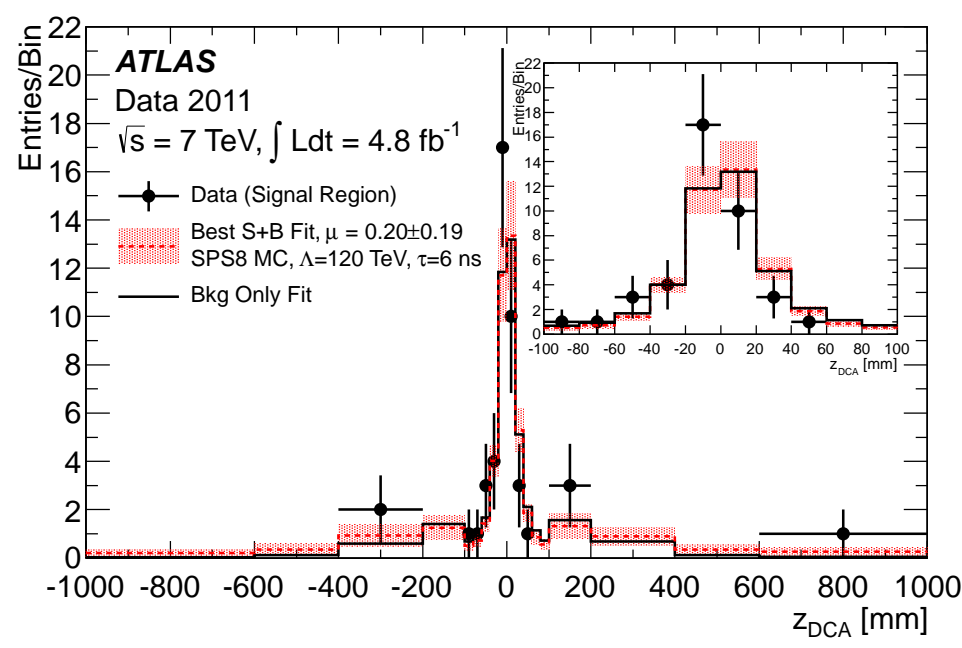

Figure 1: The $z_{D C A}$ distribution for photon candidates in the search for $\tilde{\chi}_{1}^{0} \rightarrow \gamma \tilde{G}$ decays. Superimposed are the results of the background-only fit, as well as the results of the signal-plus-background fit, shown with the associated uncertainty band.

gravitino $\left(\tilde{\chi}_{1}^{0} \rightarrow \gamma \tilde{G}\right)$ would generate events with the typical signature of two photons and missing transverse momentum. The photon from a displaced decay vertex could be distinguished from prompt photons exploiting two features: the reconstructed line of flight would not point back towards the $p p$ interaction vertex (but rather towards the displaced $\tilde{\chi}_{1}^{0}$ decay vertex) and the arrival time at the calorimeter would be delayed. Thanks to its longitudinal segmentations with small $\eta$ strips, the ATLAS liquid argon (LAr) electromagnetic calorimeter can measure the direction of the photons with good resolution. The variable used to quantify the degree of non-pointing of the photon is $z_{D C A}$, the distance along the $z$-direction between the primary interaction vertex and the point of closest approach between the beamline and the photon line of flight. The resolution on $z_{D C A}$, is abut $15 \mathrm{~mm}$ for prompt photons at central rapidity and with energy in the range $50-100 \mathrm{GeV}$. The resolution on the arrival time of the photons is approximately $0.29 \mathrm{~ns}$, mostly due to the spread of the pp collisions ( $0.22 \mathrm{~ns})$. The analysis of the $\sqrt{s}=7 \mathrm{TeV}$ data [8], corresponding to an integrated luminosity $L=4.8 \mathrm{fb}^{-1}$, is based on the selection of events with two photons with $p_{T}>50 \mathrm{GeV}$ and missing transverse momentum above $75 \mathrm{GeV}$. Tight identification requirements have been applied to one photon. A looser selection has been applied to the other photon, to avoid biases in the direction measurement. The distribution of $z_{D C A}$ of the photon with the looser selection is shown in Figure 1 for the 46 surviving events. The distribution is in good agreement with the background expectation, obtained from a mixture of $Z \rightarrow e e$ and multijet events. The timing distribution is also compatible with prompt production. This result has been used to set limits on the NLSP lifetime in a reference model known as Snowmass point SPS8. In this model, a $\tilde{\chi}_{1}^{0}$ with lifetime between 0.25 $(0.25) \mathrm{ns}$ and $50(2.7) \mathrm{ns}$ is excluded by this analysis for a scale $\Lambda=70$ (170) $\mathrm{TeV}$, corresponding to a $\tilde{\chi}_{1}^{0}$ mass of 100 (240) GeV. 


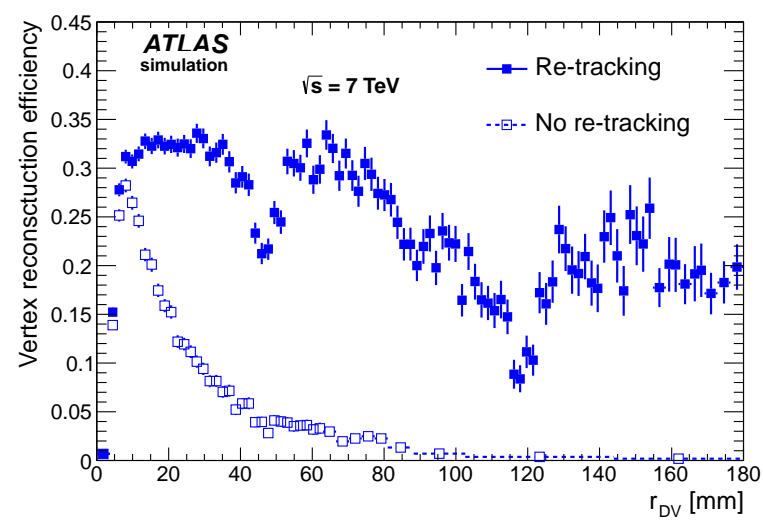

Figure 2: Vertex reconstruction efficiency as a function of the displaced vertex radius $r_{D V}$, for the standard and the dedicated reconstruction with re-tracking. The dips correspond to decays immediately before a pixel detector layer, where many tracks from the vertex have shared pixel hits failing track quality requirements.

\section{Displaced vertex plus muon}

In RPV SUSY models, the lightest neutralino could decay into a muon and two jets through the Yukawa coupling $\lambda_{2 i j}^{\prime}\left(\tilde{\chi}_{1}^{0} \rightarrow \mu q_{i} \bar{q}_{j}\right)$. The standard ATLAS tracking and vertex reconstruction algorithms are optimized for vertices produced within few $\mathrm{mm}$ from the interaction point, loosing efficiency at larger radial distance from the beamline, $r_{\mathrm{DV}}$. A dedicated algorithm has been developed to reconstruct a DV at larger $r_{\mathrm{DV}}$ in the ID, including a re-tracking step to recover hits not associated with tracks originating from the interaction point. The efficiency improvement is shown in Figure 2. A search for events with a muon and a DV has been performed on $\sqrt{s}=7 \mathrm{TeV}$ data $^{1}$ [9]. Events have been selected requiring an high- $p_{T}$ muon and a DV with $3<r_{\mathrm{DV}}<180 \mathrm{~mm}$. Only vertices located outside the material of the ID have been considered, to remove displaced vertices from secondary interaction. Finally the vertex has been required to have large charged multiplicity $\left(N_{\mathrm{DV}}>4\right)$ and high mass $\left(M_{\mathrm{DV}}>10 \mathrm{GeV}\right)$. No event has been found in the signal region, to be compared to an expected background from combinatorials and interactions with air molecules of $\left(4_{-4}^{+60}\right) 10^{-3}$ events. This result has been used to set limits on the production of squark pairs with their subsequent decay $\tilde{q} \rightarrow q \tilde{\chi}_{1}^{0}$ and $\tilde{\chi}_{1}^{0} \rightarrow \mu q_{i} \bar{q}_{j}$ through $\lambda_{2 i j}^{\prime}$. As an example, supergravity models with squark mass $m_{\tilde{q}}=700 \mathrm{GeV}$ and a neutralino masses $m_{\tilde{\chi}_{1}^{0}}=108 \mathrm{GeV}$ are excluded up to $\tilde{\chi}_{1}^{0}$ lifetimes of approximately $c \tau=1 \mathrm{~m}$, for a branching ratio of one.

\section{Stable charged particles}

In GSMB models the lightest stau $\left(\tilde{\tau}_{1}\right)$ could act as a long-lived NLSP. It would appear as a "heavy muon", passing through the ATLAS sub-detectors without showering. Due to its large mass it may have $\beta$ significantly lower than one and an energy loss by ionization higher than the minimum. A first search was performed on $\sqrt{s}=7 \mathrm{TeV}$ data [6]. The analysis has now been

\footnotetext{
${ }^{1}$ After the conference this search has been extended to $\sqrt{s}=8 \mathrm{TeV}$ data [10]
} 

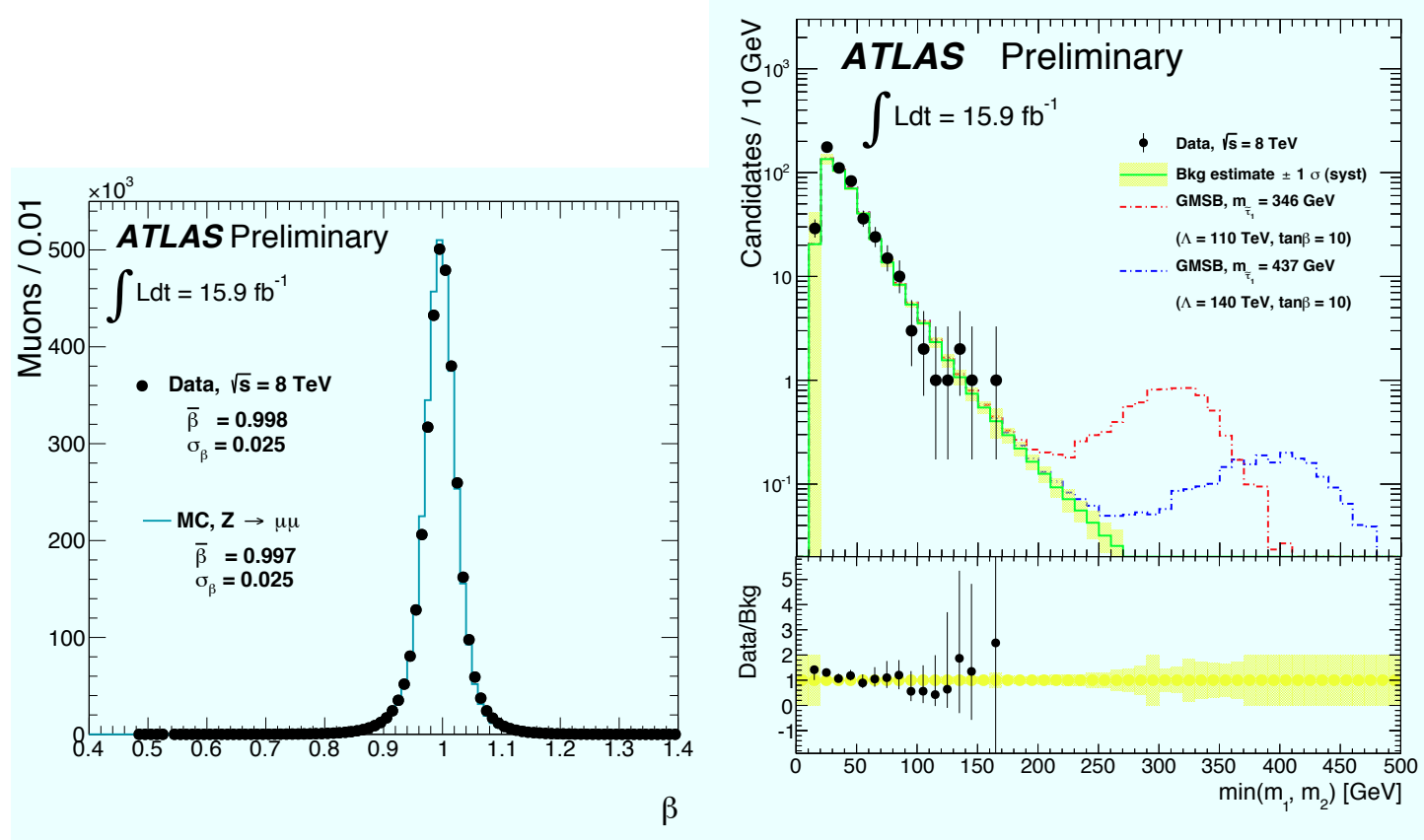

Figure 3: Left: measured velocity $\beta$ for muons from $Z$ decays. Right: distribution of the lowest reconstructed mass for events with a pair of long-lived slepton candidates, compared to the background estimate and two examples of simulated signals.

extended on $L=15.9 \mathrm{fb}^{-1}$ of $\sqrt{s}=8 \mathrm{TeV}$ data [11]. The $\tilde{\tau}_{1}$ (or other sleptons) has been sought in muon triggers. The particle track has been reconstructed in the MS using a dedicated reconstruction algorithm which fits simultaneously the track parameters and $\beta$. The $\beta$ measured in the MS has been combined with timing measurements in the calorimeters to obtain a global measurement of the particle velocity. The typical resolution on $\beta$, measured for muons from $Z$ decays, is $\sigma_{\beta}=0.025$, as shown in Figure 目(left).

The compatibility of the timing measurements from different sub-detectors and the consistency with the energy loss measured in the pixels of the ID have been used to clean the sample, leaving only tracks with a well measured mass, given by $m=p /(\beta \gamma)$. As the long-lived sleptons are expected to be produced in pairs, the highest sensitivity is obtained by searching events with two different high $p_{T}$ candidates. The main background comes from instrumental tails in the $\beta$ resolution and has been estimated by mixing $\beta$ measurements and track momenta from independent control samples. Figure 3 (right) shows the reconstructed mass of the lightest candidate for events with two candidates with $p_{T}>50 \mathrm{GeV}$ and $\beta<0.95$, compared to the expected background. No event has been observed above $m \simeq 170 \mathrm{GeV}$. The efficiency times acceptance of this selection, for events with directly produced stau pairs, is approximately $20 \%$, which gives $95 \%$ upper limits on the cross section of $\sigma_{\lim } \approx 1 \mathrm{fb}$ over the mass range $250<m_{\tilde{\tau}}<500 \mathrm{GeV}$.

Different processes would lead to the production of stau pairs: the Drell-Yan-like direct production of slepton pairs; the strong production of gluinos and squarks with subsequent cascade decays; the electrowaek production of chargino and/or neutralino pairs, with the following decay 

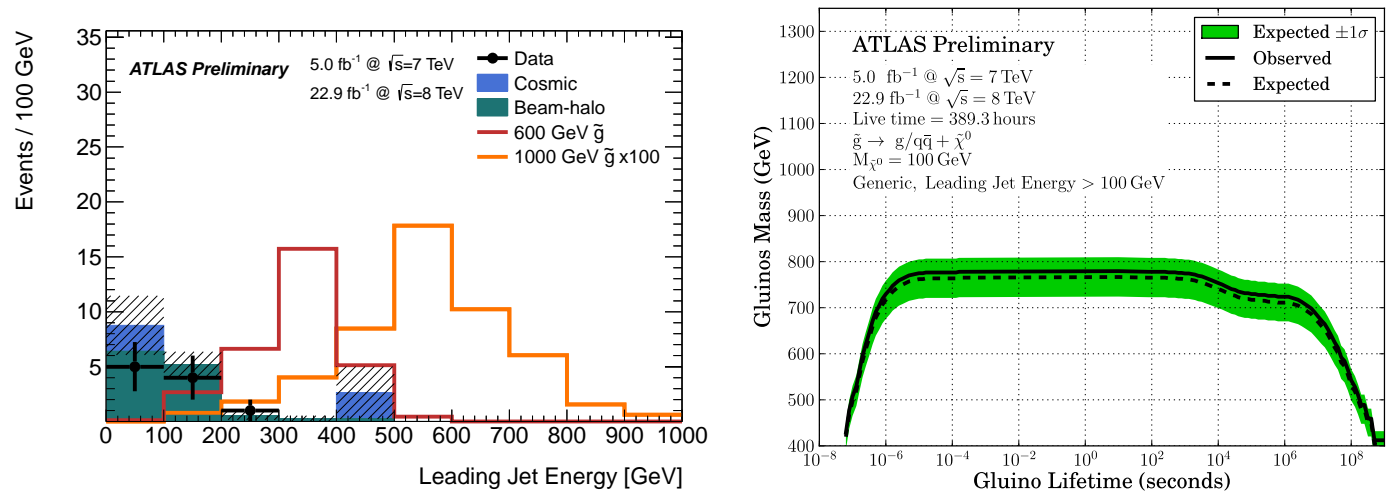

Figure 4: Left: transverse energy of the leading jet for out-of-time events, the data are compared to to background expectations from cosmic and beam-halo muons and to two simulated signals. Right: Mass limits for a gluino decaying into a $g \tilde{\chi}_{1}^{0}$ or to $q \bar{q} \tilde{\chi}_{1}^{0}$ as a function of the gluino lifetime, for a neutralino mass $M_{\tilde{\chi}_{1}^{0}}=100 \mathrm{GeV}$.

into $\tilde{\tau}_{1}$. Mass limits have been derived in the context of GSMB models with $N_{5}=3, m_{\text {messanger }}=$ $250 \mathrm{TeV}, \operatorname{sign}(\mu)=+1$. At the time of the conference, only mass limits for the electroweak neutralino and chargino production were available. They correspond to the exclusion of a $\tilde{\chi}_{1}^{0}$ decaying into $\tilde{\tau}_{1}$ for $M_{\tilde{\chi}_{1}^{0}}<475-490 \mathrm{GeV}$, depending on the $\tan \beta$ parameter. Assuming that the only production mechanism is the direct production of $\tilde{\tau}_{1}$ pairs, then $m_{\tilde{\tau}}$ is excluded below $267 \mathrm{GeV}$ at $95 \%$ CL.

\section{Out-of-time jets}

In RPV models, squarks and gluinos can be long-lived and hadronize into so-called R-hadrons. Massive R-hadrons could be detected as penetrating particles with large energy loss and low $\beta$ [6]. They could also come to rest inside the ATLAS calorimeter and decay after a significant time. This would lead to the striking signature of large energy deposits delayed with respect to the $p p$ collisions. The full available $p p$ data set $\left(L=5.3 \mathrm{fb}^{-1}\right.$ collected at $\sqrt{s}=7 \mathrm{TeV}$ and $L=22.9 \mathrm{fb}^{-1}$ collected at $\sqrt{s}=8 \mathrm{TeV}$ ) has been used to search for out-of-time jets [12]. The LHC bunch structure in 2011 and 2012 was such that more than half of the $25 \mathrm{~ns}$ intervals defined by the LHC clock did not correspond to the passage of proton bunches in the interaction point (empty bunches). A dedicated trigger was used to select events with jets and missing transverse momentum in empty bunches, for a total live time of 515.1 hours. This method gives sensitivity to R-hadron lifetimes ranging from $100 \mathrm{~ns}$ to approx. 100 days. The main backgrounds are muons from cosmic rays and from upstream beam-halo interactions. The first low-luminosity part of the data set has been used to evaluate the cosmic background, while a sample of beam-halo events has been collected in "unpaired bunches", in which only one of the two beams contained a proton bunch. A dedicated muon veto has been used to reduce the backgrounds. The leading-jet transverse energy for the final sample is shown in Figure 4 (left) compared to the expected background and examples of simulated 
signals. As there is no evidence of any excess, limits on gluino masses have been set as a function of the R-hadron lifetime. An example is given in Figure $\$$ (right).

\section{Conclusions}

The ATLAS collaboration is undertaking an ongoing effort to push the detector capabilities to detect long-lived particles and their unusual signatures. Several searches for new physics have been performed recently exploiting these capabilities: searches for long-lived neutralinos decaying into photons and gravitinos in GMSB models, for long-lived neutralinos decaying into a muon and jets in RPV models, for meta-stable sleptons predicted by GMSB models and for long-lived gluinos in RPV models. No evidence for LLPs has been found so far and the null results were used to set new stringent limits on GMSB and R-parity violating supersymmetry.

\section{References}

[1] Atlas Collaboration, JINST 3 (2008) S08003.

[2] W. Ehrenfeld, these proceedings.

[3] Atlas Collaboration, PRL 108 (2012) 251801.

[4] Atlas Collaboration, PLB 722, (2013) 305.

[5] Atlas Collaboration, PRL 109 (2012) 261803.

[6] Atlas Collaboration, PLB 720 (2013) 277.

[7] Atlas Collaboration, JINST 8 (2013) P07015.

[8] Atlas Collaboration, PRD 88, (2013) 012001.

[9] Atlas Collaboration, PLB 719 (2013) 280.

[10] Atlas Collaboration, ATLAS-CONF-2013-092 https://cds.cern.ch/record/1595755.

[11] Atlas Collaboration, ATLAS-CONF-2013-058 https://cds.cern.ch/record/1557775.

[12] Atlas Collaboration, ATLAS-CONF-2013-057 https://cds.cern.ch/record/1557774. 